\title{
Datta-Das transistor: Significance of channel direction, size dependence of source contacts, and boundary effects
}

\author{
Ming-Hao Liu and Ching-Ray Chang \\ Department of Physics, National Taiwan University, Taipei 106, Taiwan \\ (Received 20 July 2005; revised manuscript received 21 February 2006; published 2 May 2006)
}

\begin{abstract}
We analyze the spin expectation values for injected spin-polarized electrons (spin vectors) in a [001]-grown Rashba-Dresselhaus two-dimensional electron gas (2DEG). We generalize the calculation for point spin injection in semi-infinite 2DEGs to finite-size spin injection in bounded 2DEGs. Using the obtained spin vector formula, significance of the channel direction for the Datta-Das transistor is illustrated. Numerical results indicate that the influence due to the finite-size injection is moderate, while the channel boundary reflection may bring unexpected changes. Both effects are concluded to decrease when the spin-orbit coupling strength is strong. Hence [110] is a robust channel direction and is therefore the best candidate for the design of the Datta-Das transistor.
\end{abstract}

DOI: 10.1103/PhysRevB.73.205301

PACS number(s): 72.25.Dc, 71.70.Ej, 85.75.Hh

The Datta-Das spin-field-effect transistor (spin-FET), ${ }^{1}$ stimulating plenty of theoretical and experimental works in semiconductor spintronics, ${ }^{2}$ has not yet been realized. Concluded difficulties are basically: ${ }^{3}$ (i) effective controllability of the Rashba spin-orbit ${ }^{4}$ (SO) coupling strength $\alpha$, (ii) long spin-relaxation time in two-dimensional electron gas (2DEG) systems, (iii) uniformity of $\alpha$, and (iv) a more efficient spin injection rate. So far, the two former conditions have been basically satisfied in experiments, ${ }^{5,6}$ while the latter two remain to be solved.

In the original proposal of the Datta-Das spin-FET, the structure inversion asymmetry (the Rashba SO term) is required to dominate over the bulk inversion asymmetry (the Dresselhaus SO term, ${ }^{7}$ with coupling strength $\beta$ ) therein. However, the coupling strengths of the Rashba and Dresselhaus terms have been, in fact, found to be of the same order in certain types of quantum wells. ${ }^{8,9}$ Therefore, the influence due to the Dresselhaus term has become another issue in spintronics. For example, Łusakowski et al. had shown that the conductance of the Datta-Das spin-FET depends significantly on the crystallographic direction of the channel in the presence of the Dresselhaus term. ${ }^{10}$ A more complete work done by Winkler is the investigation of the spin splitting due to the effective magnetic field generated by the structure inversion asymmetry and the bulk inversion asymmetry. ${ }^{11,12}$

Recently, our previous work following Winkler even derived the analytical formulae of the electron spin precession in the 2DEG with both the Rashba and Dresselhaus terms involved. ${ }^{13}$ The formulae obtained in Ref. 13 also implies the significance of the $2 \mathrm{DEG}$ channel direction, and is therefore in correspondence with Łusakowski's result. However, the assumption of spin injection via an ideal point contact and the neglect of boundary effects in the 2DEG channel need to be further investigated. In this paper, we mainly extend our previous work $^{13}$ to include spin injection via finite-size source contacts and to take the boundary effect into account. The former consideration is found to provide an average effect and the change thus induced is moderate, while the latter may bring drastic influences. Both effects are concluded to be strong (weak) in weak (strong) SO-coupling channels. In the case of $\alpha \beta>0$, electrons encounter the strongest spinsplitting along the [110] direction, ${ }^{14}$ which is therefore concluded to be a robust channel direction as a good choice of the Datta-Das spin-FET. Throughout this paper, we work within the single-particle picture using a standard quantum mechanical approach, in particular, the time-independent Schrödinger picture, and assume zero temperature in the clean limit.

Before considering the finite-size injection contact and the boundary effect in the 2DEG channel, we first generalize the formulae obtained in Ref. 13, which mainly describes the in-plane behavior of the electron spin, injected from an inplane-magnetized ferromagnet into the 2DEG, via an ideal point contact. Referring to their results as $\langle\mathbf{S}\rangle_{\mathbf{r}}^{\|}$with the superscript denoting that the injected spin is inplane polarized while the subscript is for expectation done on $\mathbf{r}=(r, \phi)$, we are now considering the more general case, namely, spin injection with arbitrary polarization. The spinor corresponding to the electron spin injected on $\mathbf{r}_{i}$ is therefore given by ${ }^{15}$ $|\mathbf{s}\rangle_{\mathbf{r}_{i}} \doteq\left(e^{-i \phi_{s}} \cos \left(\theta_{s} / 2\right),+\sin \left(\theta_{s} / 2\right)\right)^{T}$, and we are thus seeking the spin vector $\langle\mathbf{S}\rangle_{\mathbf{r}}=(\hbar / 2)\langle\vec{\sigma}\rangle_{\mathbf{r}}$ with $\vec{\sigma}$ being the Pauli matrices. Also, we present the calculation of $\left\langle\sigma_{z}\right\rangle_{\mathbf{r}}$ to complete the description of the spatial behavior of the spin vector. Using the same method introduced in Ref. 13, we obtain, choosing $\mathbf{r}_{i}=\mathbf{0}$,

$$
\langle\vec{\sigma}\rangle_{\mathbf{r}}=\left(\begin{array}{c}
-\cos \theta_{s} \cos \varphi \sin \Delta \theta(\mathbf{r})+\sin \theta_{s}\left\langle\sigma_{x}\right\rangle_{\mathbf{r}}^{\|} \\
-\cos \theta_{s} \sin \varphi \sin \Delta \theta(\mathbf{r})+\sin \theta_{s}\left\langle\sigma_{y}\right\rangle_{\mathbf{r}}^{\|} \\
\cos \theta_{s} \cos \Delta \theta(\mathbf{r})+\sin \theta_{s}\left\langle\sigma_{z}\right\rangle_{\mathbf{r}}^{\|}
\end{array}\right)
$$

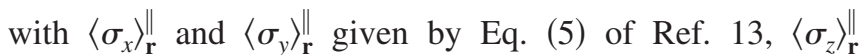
$=\cos \left(\varphi-\phi_{s}\right) \sin \Delta \theta, \quad \varphi \equiv \arg [(\alpha \cos \phi+\beta \sin \phi)+i(\alpha \sin \phi$ $+\beta \cos \phi)]$, and $\Delta \theta(\mathbf{r})=2 m^{*} r \sqrt{\alpha^{2}+\beta^{2}+2 \alpha \beta \sin (2 \phi)} / \hbar^{2}$ with $m^{*}$ the electron effective mass, for the point spin injection case in the absence of boundary effects. Clearly, Eq. (1) recovers the previous results in Ref. 13 when putting $\theta_{s}=\pi / 2$.

Next we consider a spin-polarized source connected to the 2DEG channel, either from the side or from the top, via a 
finite-size contact. Assume that each electron is equally likely to be injected via all the possible injection points, which may be everywhere on the contact except the positions close to the atoms. Let us assume that the possible injection points are located on exactly the center of each primitive unit cell of the contact crystal for the top injection. In the case of side injection, the contact region becomes a line and the injection points are reduced to the middle points of each neighboring pair of atoms. Note that despite a displacement, the distribution of the injection points are equivalent to the lattice points of the contact. Labeling the positions of the injection points as $\mathbf{r}_{i}$, the state ket describing the injected electron detected on $\mathbf{r}$ may be superposed by $\left.|\mathbf{s}\rangle_{\mathbf{r}}=(1 / \mathbf{r}|\mathbf{s}| \mathbf{s}\rangle_{\mathbf{r}}\right) \sum_{i}|\mathbf{s}\rangle_{\mathbf{r}_{i} \rightarrow \mathbf{r}}$ with $|\mathbf{s}\rangle_{\mathbf{r}_{i} \rightarrow \mathbf{r}}$ given by ${ }^{13}$ $|\mathbf{s}\rangle_{\mathbf{r}_{i} \rightarrow \mathbf{r}}=\Sigma_{\sigma= \pm 1} \exp \left[-i \sigma \Delta \theta\left(\mathbf{r}-\mathbf{r}_{i}\right) / 2\right]\left\langle\psi_{\sigma} ; \phi \mid \mathbf{s}\right\rangle_{\mathbf{r}_{i}}\left|\psi_{\sigma} ; \phi\right\rangle$, where $\left|\psi_{\sigma} ; \phi\right\rangle$ is the eigenspinor of the Rashba-Dresselhaus system, $\phi$ being the angle of the wave vector. The subscript $\mathbf{r}_{i} \rightarrow \mathbf{r}$ is to remind one that the spin is injected on $\mathbf{r}_{i}$ and detected on $\mathbf{r}$ after straight evolution. Note that this formulation can also include the problem of imperfect spin-polarized injection, i.e., to deal with a multidomain ferromagnetic source contact.

Now we deal with the channel boundary. The effect of the lateral confinement in the channel was previously regarded as to provide a large energy gap between two neighboring subbands to avoid intersubband mixing in the transverse direction. ${ }^{1,16,17}$ Contrary to this suggested quasi-onedimensional channel, we study a fully two-dimensional channel and put emphasis on the electron wave property, i.e., both longitudinal and transverse directions are not strongly quantized, so that the spatial parts of the electron wave function in both directions are described by plane waves (under the effective mass Hamiltonian). Based on this viewpoint, we assume that each spin-polarized electron injected on $\mathbf{r}_{i}$ may spatially evolve to the detection point $\mathbf{r}$ through not only the straight but also the reflected paths. Treating the lateral boundaries as hard walls, we write the corresponding state ket as

$$
|\mathbf{S}\rangle_{\mathbf{r}_{i} \rightarrow \mathbf{r}}^{\mathrm{ref}}=\frac{1}{\operatorname{ref}_{\mathbf{r}_{i} \rightarrow \mathbf{r}}\langle\mathbf{s} \mid \mathbf{s}\rangle_{\mathbf{r}_{i} \rightarrow \mathbf{r}}^{\mathrm{ref}}} \sum_{n=0}^{\infty}|\mathbf{s}\rangle_{\mathbf{r}_{i} \rightarrow \mathbf{r}}^{(n)},
$$

where $|\mathbf{s}\rangle_{\mathbf{r}_{i} \rightarrow \mathbf{r}}^{(n)}$ is the spatially evolved state ket from $\mathbf{r}_{i}$ to $\mathbf{r}$ after $n$ times of reflection by the channel boundary. To avoid complicating the problem, we will pick terms up to $n=1$ in the numerical results for the spin vectors under the influence of boundary effects. Note that the source and drain contacts are assumed to be ohmic, and hence we neglect the reflections in the longitudinal direction.

Note that to obtain the reflected waves is somewhat tricky. For example, the $n=1$ term with $\mathbf{r}_{i} \rightarrow \mathbf{r}^{\prime} \rightarrow \mathbf{r}$ path, where $\mathbf{r}^{\prime}$ is the position vector where the reflection occurs, can be obtained by $|\mathbf{s}\rangle_{\mathbf{r}_{i} \rightarrow \mathbf{r}^{\prime} \rightarrow \mathbf{r}}$ $=\Sigma_{\sigma} \exp \left[-i \sigma \Delta \theta\left(\mathbf{r}-\mathbf{r}^{\prime}\right) / 2\right]\left\langle\psi_{\sigma} ; \phi_{\mathbf{r}-\mathbf{r}^{\prime}} \mid \mathbf{s}\right\rangle_{\mathbf{r}_{i} \rightarrow \mathbf{r}^{\prime}}\left|\psi_{\sigma} ; \phi_{\mathbf{r}-\mathbf{r}^{\prime}}\right\rangle^{i}$ with $|\mathbf{s}\rangle_{\mathbf{r}_{i} \rightarrow \mathbf{r}^{\prime}}=\Sigma_{\sigma} \exp \left[-i \sigma \Delta \theta\left(\mathbf{r}^{\prime}-\mathbf{r}_{i}\right) / 2\right]\left\langle\psi_{\sigma} ; \phi_{\mathbf{r}^{\prime}-\mathbf{r}_{i}} \mid \mathbf{s}\right\rangle_{\mathbf{r}_{i}}\left|\psi_{\sigma} ; \phi_{\mathbf{r}^{\prime}-\mathbf{r}_{i}}\right\rangle$, $\phi_{\mathbf{r}}$ being the argument of the vector $\mathbf{r}$. When considering finite-size injection, the total state ket characterizing the in-

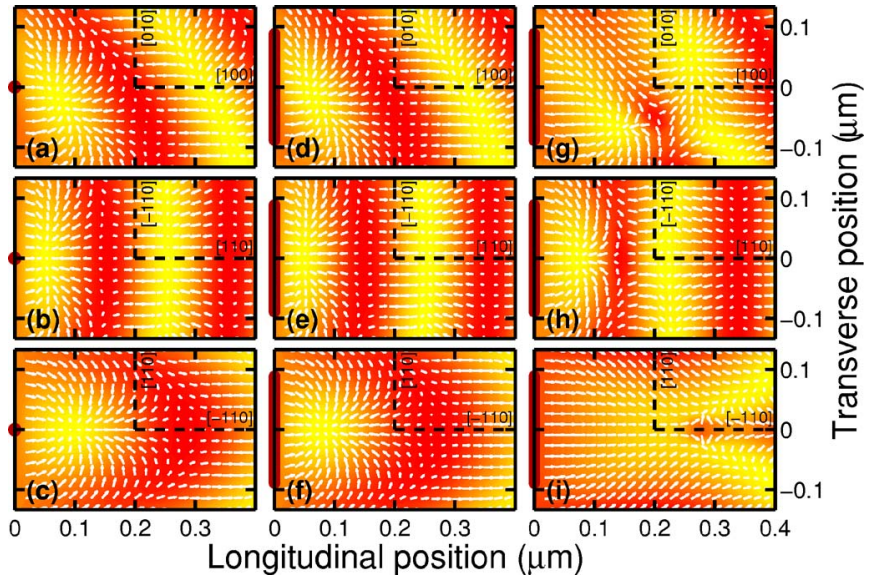

FIG. 1. (Color online) Spin vectors in $0.4 \mu \mathrm{m} \times 0.267 \mu \mathrm{m}$ InGaAs 2DEG channels using (a)-(c) point spin injection and (d)(i) finite-size spin injection, with the source contacts (indicated by dark dots or dark thick lines in each panel) all polarized parallel to the channel directions. Channel boundary effect is considered in (g)-(i). Color shading is determined by $\left\langle S_{z}\right\rangle$ with red (dark) $\rightarrow$ negative and yellow (bright) $\rightarrow$ positive.

jected electron is expressed as $|\mathbf{s}\rangle_{\mathbf{r}}=\left(1 /{ }_{\mathbf{r}}\langle\mathbf{s} \mid \mathbf{s}\rangle_{\mathbf{r}}\right) \sum_{i}|\mathbf{s}\rangle_{\mathbf{r}_{i} \rightarrow \mathbf{r}}^{\mathrm{ref}}$ with $|\mathbf{s}\rangle_{\mathbf{r}_{i} \rightarrow \mathbf{r}}^{\mathrm{ref}}$ given by Eq. (2).

Now we present the calculated spin vectors inside the 2DEG channel with certain cases of spin injection. We investigate InGaAs 2DEG channels, setting the Rashba coupling parameter $^{18} \quad \alpha=0.3 \mathrm{eV} \AA$ with electron effective mass $m^{*}=0.03 m_{e}$, in the spin transistor geometry, i.e., injected spin-polarization parallel to the channel direction. The Dresselhaus coupling parameter is chosen as $\beta=0.09 \mathrm{eV} \AA$, which is deduced from $\beta \approx \gamma\left\langle k_{z}^{2}\right\rangle$ with a typical value for the coefficient $^{8,19} \gamma \approx 25 \mathrm{eV}^{3}$, assuming an infinite quantum well in the $z$ direction with a well width $50 \AA$.

We begin with the single-point spin injection case for different channel directions without boundary effects. Three $0.4 \mu \mathrm{m} \times 0.267 \mu \mathrm{m}$ 2DEG channels along [100], [110], and [110] are examined, and the spin is injected on the middle point of the left end. Using Eq. (1), we sketch the spin vectors inside the three channels in Figs. 1(a)-1(c). Different spin patterns shown in the three cases indicate that the $z$-rotational symmetry is broken due to the presence of the Dresselhaus term. Thus the influence due to the bulk inversion-asymmetry is clear, even in this Rashba dominating 2DEG. As suggested in our previous work, ${ }^{13}$ channel directions should be chosen along [1 $1 \pm 10]$ since the Rashba and Dresselhaus terms generate a $k$-dependent effective magnetic field, which is perpendicular to the electron propagation only along these two directions. Such a uniqueness of these two axes we have just shown also agrees with Averkiev's conclusion that $[1 \pm 10]$ are the principle axes of the spin relaxation rate tensor. ${ }^{20}$

We now consider finite-size spin injection. The width of the contact is set $2 / 3$ times the channel width, and perfect polarization of the source contact in the spin transistor geometry is assumed. The spin vectors are plotted in Figs. 1(d)-1(i), where the middle and right columns are in the absence and in the presence of the boundary effect, respec- 


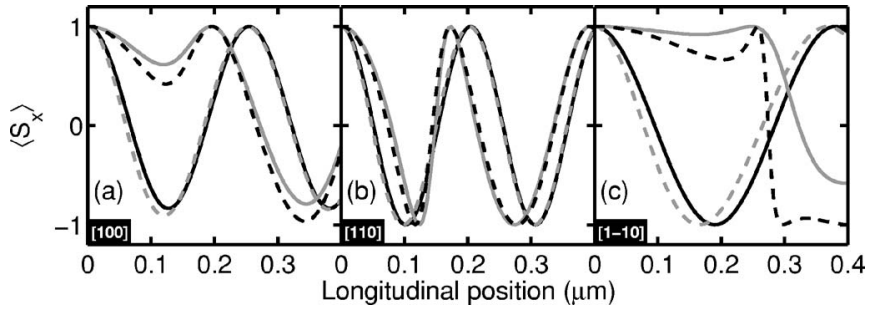

FIG. 2. The $x$-component $\left\langle S_{x}\right\rangle$ in units of $\hbar / 2$ along the straight path in the middle of a (a) [100], (b) [110], and (c) [1ㅜㅣ] channel. Legend: black solid and gray dashed lines depict single-point and finite-size injection cases, respectively, without the boundary effect; gray solid and black dashed lines depict single-point and finite-size injection cases, respectively, in the presence of the boundary effect.

tively. Compared to the single-point injection shown in Figs. 1(a)-1(c), the variation due to the finite-size spin injection seems tiny in the case without the boundary effect [Figs. 1(d)-1(f)], and the assumption of single-point injection may thus work well in this case. When the boundary effect is taken into account, the spin vectors are drastically changed [Figs. 1(g)-1(i)]. Comparing Figs. 1(d)-1(f) with 1(g)-1(i), respectively, one can roughly conclude that the influence due to the boundary effect is stronger (weaker) in weaker (stronger) SO-coupling channels, recalling that this spin-splitting is strongest along [110] whereas that along $[1 \overline{1} 0]$ is weakest when $\alpha \beta>0$. In fact, this is also true for the influence due to the finite-size injection, as will be clearer later.

To specify the change due to the finite-size spin injection and the channel boundary effect, we analyze $\left\langle S_{x}\right\rangle$ along the straight paths in the middle of each channel, as shown in Fig. 2. Without boundary effect, the effect of the finite-size injection merely reduces the spin precession lengths near the source contacts, and the corresponding reduction seems clearest and vaguest in the weakest $([1 \overline{1} 0])$ and strongest ([110]) SO-coupling channels, respectively. This behavior becomes even clearer when the boundary effect is present. Comparing black dashed (finite-size injection) with gray solid lines (point injection), the difference seems, again, clearest (vaguest) in the $[1 \overline{1} 0]$ ([110]) channel. When focusing individually on the effect of the boundary reflection, similar behavior is observed. Taking the single-point injection cases (black and gray solid lines in Fig. 2) for illustration, the change due to the boundary effect appears again the most drastic in the case of [1히, where the spin precession behavior is almost destroyed.

Moreover, such interference effect may grow with the increase of the channel width. Figure 3 shows $\left\langle S_{z}\right\rangle$ under the influence of the boundary effect and under the same conditions with Fig. 2, except the varying channel widths. Clearly, we see that the interference effect grows fastest with the increase of the channel width in the [1 $\overline{1} 0]$ channel, while the most slowly in the [110] channel, implying its robustness. Also, the precession behavior is destroyed when the channel width is wide enough. This conclusion that when one considers wider (narrower) channels, the influence of the boundary effect becomes stronger (weaker), also agrees with the

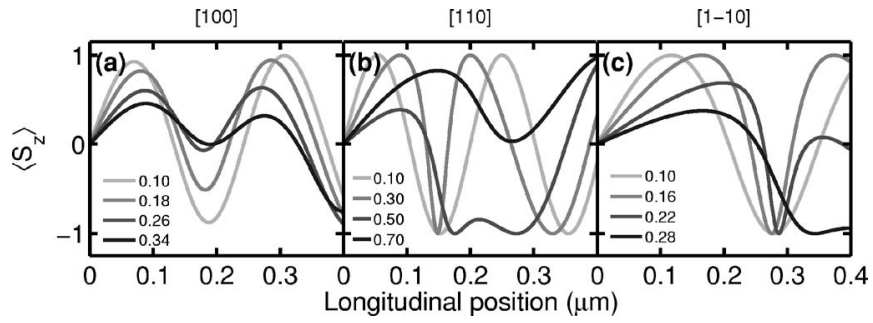

FIG. 3. The $z$-component $\left\langle S_{z}\right\rangle$ in units of $\hbar / 2$ along the straight path in the middle of a (a) [100], (b) [110], and (c) [1 10] channel with different channel widths. The legends in each panel label the corresponding channel widths in units of $\mu \mathrm{m}$.

previous suggestions of using a quasi-one-dimensional channel for enhancing the performance of the Datta-Das transistor, ${ }^{16}$ and also the slowdown of the D'yakonov-Perel' spin relaxation rate in narrow channels. ${ }^{21}$

We finally make a simple connection to the ballistic spin transport, solving for the transmission problem in a ferromagnet-2DEG-ferromagnet double junction structure, constructed by Matsuyama et al. ${ }^{22}$ who considered only the Rashba term in the 2DEG channel. As shown in Fig. 4(a), we analyze the in-plane components of the spin vectors in a $150 \mathrm{~nm} \times 1.0 \mu \mathrm{m}$ Rashba-type 2DEG (for consistency with their work), using the method of single-point injection.

From the time-independent Schrödinger equation, one can obtain the corresponding transmission probabilities, which are found to depend on (i) the Fermi velocity mismatch between the ferromagnet and 2DEG regions and (ii) the spinor overlap between the incoming and outgoing states. Of particular interest is that the transmission probabilities (and, in fact, also the reflection probabilities) are proportional to this spinor overlap. Put in another way, the electron dislikes changing its spin direction when crossing the boundary be-

(a)

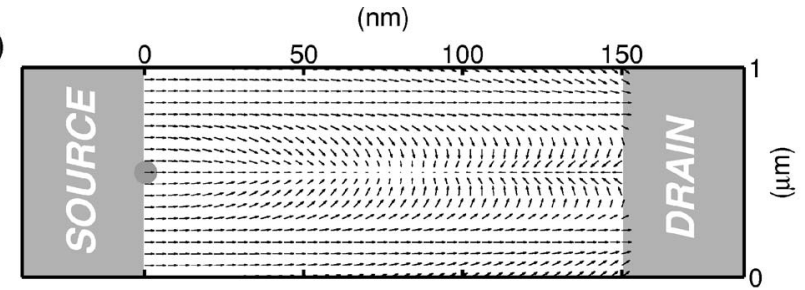

(b)

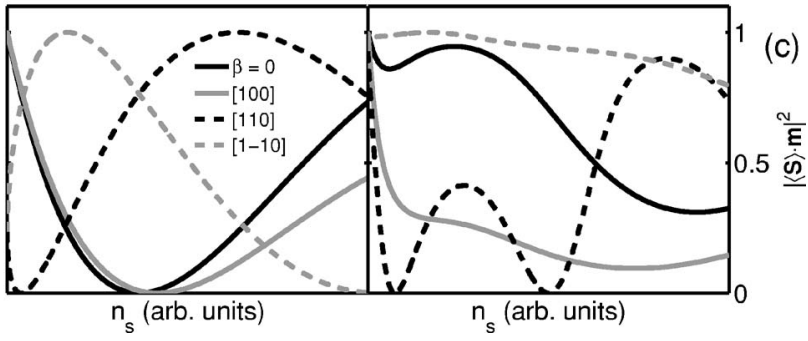

FIG. 4. (a) Spin vectors inside a Rashba-type 2DEG channel of the spin-FET. Electron spins are injected via the middle point of the source contact. (b) and (c) plot $|\langle\mathbf{S}\rangle \cdot \mathbf{m}|^{2}$ as a function of the carrier density $n_{s}$ of the 2DEG, determined on the middle point at the end of the channel in the absence and in the presence of the boundary effect, respectively. The ranging of the carrier density $n_{s}$ correspond to $\alpha=0-0.22 \mathrm{eV} \AA$. 
tween the ferromagnet and 2DEG regions. In this sense, one can clearly see why the oblique injections contribute an "undesired background" to the transmission probability, and hence the conductance, ${ }^{22}$ by noting that the spin vectors at the right end of the channel in Fig. 4(a) are nonuniform. Assuming that the drain contact is polarized parallel to the channel direction, then only the spin vectors pointing to right on the 2DEG-drain interface give a positive contribution to the conductance. Since only the normally injected spins in this Rashba channel encounter the precession axis parallel to the 2DEG-drain interface, giving rise to the maximum oscillating amplitude when varying the $\alpha$, the oscillation behavior of the total conductance obtained by summing all the transmission amplitudes from all the transmission modes (Landauer formula) will eventually be averaged down.

Focusing on the normal injection [center path in the channel of Fig. 4(a)], the spin vectors indeed precess upright down the way to the drain, as the original design of the Datta-Das spin-FET. ${ }^{1}$ Using the relation ${ }^{22} n_{s} \propto \alpha^{2}$, we plot $|\langle\mathbf{S}\rangle \cdot \mathbf{m}|^{2}(\langle\mathbf{S}\rangle$ is determined at right end of the channel, and $\mathbf{m}$ is the unit vector of the channel direction), which is responsible for the transmission probabilities $T_{ \pm, \pm}$, as a function of the carrier density $n_{s}$ in Figs. 4(b) and 4(c). In the absence the boundary effect [Fig. 4(b)], the squared projection $|\langle\mathbf{S}\rangle \cdot \mathbf{m}|^{2}$ is isotropic and shows no dependence on the crystallographic direction, when the Dresselhaus term is not involved. Despite the rapid oscillations caused by the FabryPerot interference between the source-2DEG and 2DEGdrain boundaries, we obtain a satisfactory curve [black solid line in Fig. 4(b)], in good agreement with Ref. 22 [see Figs. 10(c)-10(f) therein]. We again stress the importance of choosing the channel direction for the Datta-Das transistor with $\beta \neq 0$, by noting that the precession behavior of the injected spin is sensitive to the channel direction [the other three lines in Fig. 4(b)]. When the boundary effect is involved [Fig. 4(c)], the spin precession behavior is totally changed, even the robust [110] channel. This is because the channel width is too wide, allowing a much more severe influence caused by the boundary reflection, as we have discussed previously.

In conclusion, we have calculated the spin vectors inside the 2DEG channel of the Datta-Das transistor to demonstrate the significance of the channel direction and to investigate the size-dependence of source contacts and the channel boundary effects. The analytical spin vector formulae for the point spin injection ${ }^{13}$ are also generalized to the arbitrary polarization of spin injection cases. Numerical results have shown that the influence due to the finite-size injection is moderate, while the channel boundary reflection may bring unexpected changes.

We emphasize here the two-dimensional wave property of the electron in typical InGaAs 2DEGs. From degenerate perturbation theory, one is led to the criterion ${ }^{1,16} W \ll \hbar^{2} / \alpha m^{*}$, within which the channel can be regarded as quasi-onedimensional, when assuming hard wall lateral confinement and considering only the Rashba term. For the RashbaDresselhaus 2DEGs with, e.g., $\alpha=0.3 \mathrm{eV} \AA, \beta=0.09 \mathrm{eV} \AA$, and $m^{*}=0.03 m_{e}$, the total coupling strength ranges from $0.21 \mathrm{eV} \AA$ (the $[1 \overline{1} 0]$ direction) to $0.39 \mathrm{eV} \AA$ (the [110] direction). The criterion for the quasi-one-dimensional channel will require $W \ll 65 \mathrm{~nm}(121 \mathrm{~nm})$ for the $[110]([1 \overline{1} 0])$ case. Therefore, typical InGaAs 2DEGs with channel widths of the order of or larger than these lengths will require a twodimensional description for the electron waves, and the possible boundary effects are thus unavoidable.

Our results may be taken as a warning indicating another difficulty inherent in the design of the Datta-Das spin-FET: the interference due to the channel boundaries. However, we conclude that the [110] direction is shown to be robust under the influence of finite-size spin injection and the boundary reflection, for [001]-grown zincblend-based 2DEGs.

This work was supported by the Republic of China National Science Council Grant No. 94-2112-M-002-004.
${ }^{1}$ S. Datta and B. Das, Appl. Phys. Lett. 56, 665 (1990).

${ }^{2}$ Semiconductor Spintronics and Quantum Computation, edited by D. D. Awschalom, D. Loss, and N. Samarth (Springer, Berlin, 2002).

${ }^{3}$ Igor Žutić, Jaroslav Fabian, and S. Das Sarma, Rev. Mod. Phys. 76, 323 (2004).

${ }^{4}$ E. I. Rashba, Sov. Phys. Solid State 2, 1109 (1960); Yu. A. Bychkov and E. I. Rashba, JETP Lett. 39, 78 (1984).

${ }^{5}$ J. M. Kikkawa and D. D. Awschalom, Phys. Rev. Lett. 80, 4313 (1998).

${ }^{6}$ J. Nitta, T. Akazaki, H. Takayanagi, and T. Enoki, Phys. Rev. Lett. 78, 1335 (1997).

${ }^{7}$ G. Dresselhaus, Phys. Rev. 100, 580 (1955).

${ }^{8}$ B. Jusserand, D. Richards, G. Allan, C. Priester, and B. Etienne, Phys. Rev. B 51, R4707 (1995).

${ }^{9}$ W. Knap, C. Skierbiszewski, A. Zduniak, E. Litwin-Staszewska, D. Bertho, F. Kobbi, J. L. Robert, G. E. Pikus, F. G. Pikus, S. V. Iordanskii, V. Mosser, K. Zekentes, and Yu. B. Lyanda-Geller,
Phys. Rev. B 53, 3912 (1996); J. B. Miller, D. M. Zumbühl, C. M. Marcus, Y. B. Lyanda-Geller, D. Goldhaber-Gordon, K. Campman, and A. C. Gossard, Phys. Rev. Lett. 90, 076807 (2003); S. D. Ganichev, V. V. Bel'kov, L. E. Golub, E. L. Ivchenko, P. Schneider, S. Giglberger, J. Eroms, J. De Boeck, G. Borghs, W. Wegscheider, D. Weiss, and W. Prettl, ibid. 92, 256601 (2004).

${ }^{10}$ A. Łusakowski, J. Wróbel, and T. Dietl, Phys. Rev. B 68, 081201(R) (2003).

${ }^{11} \mathrm{R}$. Winkler, Spin-Orbit Coupling Effects in Two-Dimensional Electron and Hole Systems (Springer, Berlin, 2003).

${ }^{12}$ R. Winkler, Phys. Rev. B 69, 045317 (2004).

${ }^{13}$ M.-H. Liu, C.-R. Chang, and S.-H. Chen, Phys. Rev. B 71, 153305 (2005).

${ }^{14}$ J. Kainz, U. Rössler, and R. Winkler, Phys. Rev. B 68, 075322 (2003).

${ }^{15}$ J. J. Sakurai, Modern Quantum Mechanics, revised ed. (AddisonWesley, New York, 1994). 
${ }^{16}$ F. Mireles and G. Kirczenow, Phys. Rev. B 64, 024426 (2001).

${ }^{17}$ J. Wang, H. B. Sun, and D. Y. Xing, Phys. Rev. B 69, 085304 (2004).

${ }^{18}$ Y. Sato, T. Kita, S. Gozu, and S. Yamada, J. Appl. Phys. 89, 8017 (2001).

${ }^{19}$ G. Lommer, F. Malcher, and U. Rössler, Phys. Rev. Lett. 60, 728 (1988); B. Jusserand, D. Richards, H. Peric, and B. Etienne, ibid. 69, 848 (1992).

${ }^{20}$ N. S. Averkiev and L. E. Golub, Phys. Rev. B 60, 15582 (1999).

${ }^{21}$ A. G. Mal'shukov and K. A. Chao, Phys. Rev. B 61, R2413 (2000).

${ }^{22}$ T. Matsuyama, C.-M. Hu, D. Grundler, G. Meier, and U. Merkt, Phys. Rev. B 65, 155322 (2002). 